\title{
Continuum and discrete excitation spectrum of single quantum rings
}

\author{
Benito Alén, ${ }^{1,2, *}$ Juan Martínez-Pastor, ${ }^{1}$ Daniel Granados, ${ }^{2}$ and Jorge M. García ${ }^{2}$ \\ ${ }^{1}$ Instituto de Ciencia de los Materiales de la Universidad de Valencia, P.O. Box 22085, 46071 Valencia, Spain \\ ${ }^{2}$ Instituto de Microelectrónica de Madrid (CNM, CSIC), Isaac Newton 8, 28760 Tres Cantos (Madrid), Spain
}

(Received 14 June 2005; revised manuscript received 12 September 2005; published 27 October 2005)

\begin{abstract}
Photoluminescence and excitation of the photoluminescence spectroscopy has been performed in single InGaAs self-assembled quantum rings embedded in a field effect structure device. To determine their electronic structure, bias-dependent optical transitions have been analyzed both, for individual quantum rings, and for the averaged ensemble. Our results are compared with a theoretical model, and also with results reported by other authors studying similar nanostructures.
\end{abstract}

DOI: 10.1103/PhysRevB.72.155331

PACS number(s): 73.63.Kv, 81.07.Ta, 78.67.Hc

\section{INTRODUCTION}

The electronic and optical properties of self-assembled quantum dots (QDs) have been intensively studied in recent years, attending to both their collective behavior in ensemble averaged experiments and their individual properties using single nanostructure spectroscopy. ${ }^{1,2}$ The studies reveal that an atomiclike density of states is well suited to describe the ground and first excited states of these nanostructures. In such situations, the creation and anhilation of multiparticle exciton complexes are dominated by the Coulomb blockade and the Pauli exclusion principle, bringing the direct and exchange Coulomb interaction energies much larger than typical homogeneous linewidths in the system. ${ }^{2}$ A discrete electronic structure is necessary to allow for coherent control of individual spin and/or charge states and to fulfill the requirements of quantum information applications. ${ }^{3,4}$ Indeed, simultaneous optical and electrical access to single QDs have been demonstrated and successfully applied to implement solid-state-based quantum gates, ${ }^{5}$ or to fabricate devices such as single photon emitters. ${ }^{6}$

In spite of this success, the artificial atom picture overlooks many properties inherited by the QD from its embedding semiconductor matrix. The interaction between the discrete QD band structure and the different matrix-related continua is responsible for a number of electronic and optical properties that are not simple extrapolation of a discrete density of states. Bound to continuum states hybridization has to be considered to explain unexpected spectral features observed in recent experiments performed in single QDs. ${ }^{7,8} \mathrm{Re}-$ garding the QD spin dynamics, it has been recently shown that the interaction between localized excitons and an adjacent Fermi sea plays a major role in the exciton recombination path trace. ${ }^{9}$ Finally, strong coupling between excitons and phonons could explain the efficient carrier relaxation observed in these nanostructures and therefore a revision of the QD electronic structure and dynamics could be necessary. ${ }^{10}$

In order to establish a complete and unified picture, systematic studies based in nanostructures with different size, shape, and composition are desirable. Like QDs, selfassembled quantum rings (QRs) confine the electrical carriers in all three spatial directions and therefore are similarly characterized by a discrete density of states. However, QRs have attracted the interest of the scientific community also because of their special topology. ${ }^{1-17}$ The quantum mechanical properties of an electron system circumventing a sufficiently small quantum ring are periodic in the magnetic field flux penetrating the system. It is the signature of the Aharonov-Bohm (AB) effect. One consequence is the stepwise change in the angular momentum of the ground state observed by intraband optical and capacitance magnetodependent spectroscopy in negatively charged selfassembled QRs. ${ }^{2}$ Using interband optical excitations, the quantum interference giving rise to these phenomena is smeared out due to the neutral character of the formed exciton. ${ }^{18-21}$ Although a finite contribution has been calculated for one-dimensional QRs, ${ }^{18}$ in experiments, excitonic $\mathrm{AB}$ oscillations have been observed only in negatively charged excitons confined in lithographically defined QRs, ${ }^{22}$ whereas such effect has not been observed in similar experiments performed in self-assembled QRs. ${ }^{14} \mathrm{~A}$ better scenario has been predicted for excited states which are more delocalized along the ring perimeter, ${ }^{18,20,21}$ yet the experimental investigation of excited states in QRs is not enough to conclude any assessment in that direction. ${ }^{15,16}$ At $B=0$, the single particle $Q R$ electronic structure is determined by its shape and composition. Realistic models have been drawn taking into account the true 3D confinement geometry, ${ }^{23,24}$ and additional refinements such as strain ${ }^{25,26}$ and piezoelectric fields ${ }^{25}$ or valence band mixing contributions. ${ }^{27}$ This notwithstanding, several authors ${ }^{12,19,21,28-30}$ have achieved considerable success catching only the overall ring potential geometry with 2D paraboliclike models, but at the expense of some fitting of the model-dependent parameters to the experimental data.

In this work, we present results of microphotoluminescence ( $\mu$-PL) and excitation of the microphotoluminescence ( $\mu$-PLE) to study the electronic structure of self-assembled QRs above their ground state at zero magnetic field. In light of these results, we investigate the relative importance of the 3D confinement over the particular ring shape, and the role played by the embedding semiconductor matrix on the electronic properties of this system.

\section{SAMPLES AND EXPERIMENT}

The sample studied here contains InGaAs QRs obtained by molecular beam epitaxy partially overgrowing with GaAs 

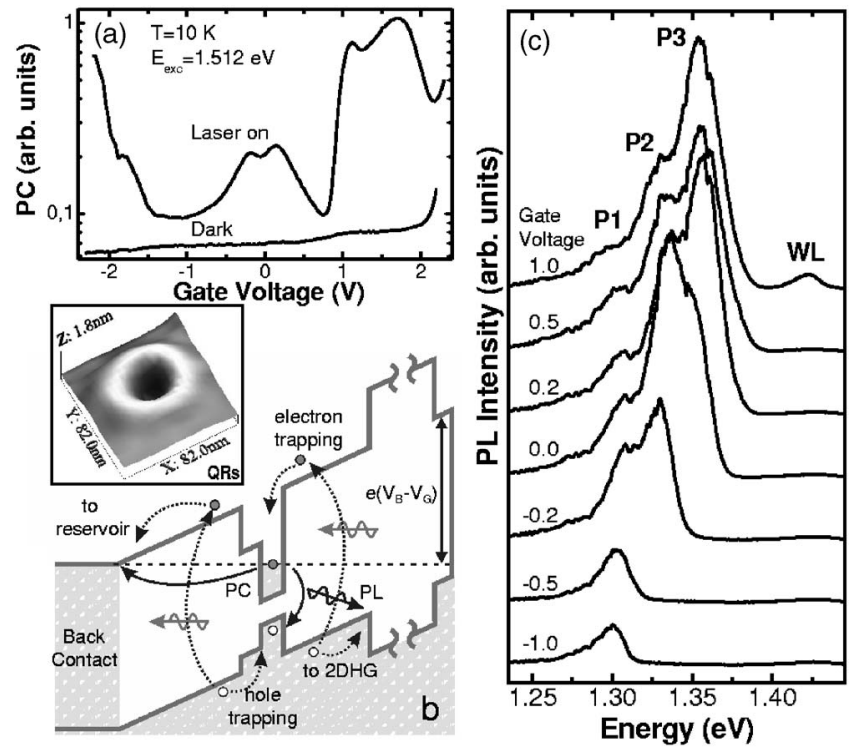

FIG. 1. (a) Semilogartithmic $I / V$ characteristic of the device in darkness and under illumination above the GaAs edge as obtained at $10 \mathrm{~K}$. (b) The band diagram in the vicinity of the active layer is shown for an externally applied reverse bias $V_{G}$. A typical AFM image of a QR is also shown in the inset. (c) Ensemble-averaged PL spectra as a function of $V_{G}$ obtained at $10 \mathrm{~K}$ and low excitation powers.

a single layer of self-assembled InAs QDs. ${ }^{17}$ Recently, cross sectional scanning tunneling microscopy (xSTM) images obtained in similar samples have been available revealing that the ring-shaped structure is preserved after capping, although its morphology does not directly reflect what we observe in uncapped samples. ${ }^{16,31}$ A hard InAs inner core $(\sim 20 \mathrm{~nm}$ diameter, $\sim 8 \mathrm{~nm}$ width) is surrounded by an alloyed Ga-rich region with a much bigger lateral extension $\sim 80 \mathrm{~nm}$. The large diameter observed in atomic force microscopy (AFM) for this kind of QRs [see the inset of Fig. 1(b)] is therefore related to the alloyed region and not to the InAs nucleus where the carriers are confined. The central hole itself, with a diameter of $\sim 12 \mathrm{~nm}$, is alloyed to a certain extent due to the incomplete dewetting process, ${ }^{17}$ and looks more like a depression rather than a hole in the XSTM images. ${ }^{16,31}$ As a result, we do not expect a hard wall potential in the center of the QR, a fact that has to be taken into account when discussing its electronic properties. ${ }^{19,29,30}$ Despite the complex in-plane morphology, the ground state energy is primarily determined by the vertical confinement. In our case, with an average height $\sim 3.5 \mathrm{~nm}$, the QR PL band reaches its maximum at $\sim 1.3 \mathrm{eV}(4.2 \mathrm{~K})$ like lens-shaped InGaAs QDs of similar dimensions.

Additional control can be achieved by embedding the QRs in a field effect structure device. In our case, the layer containing the nanostructures is separated by a 25 -nm-thick GaAs tunneling barrier from a highly doped n-type GaAs back contact. After burying the QRs with GaAs, the device is completed with a short period AlAs/GaAs superlattice (SPSL) and a semitransparent surface gate electrode (Au:Cr Schottky on GaAs) being the total distance between the active layer and the sample surface of $150 \mathrm{~nm}$.
To access the individual properties of single QRs we used a cryogenic confocal microscope based in a single mode fiber acting simultaneously as excitation and collection pinhole. The sample space numerical aperture (NA) is 0.65 , allowing for an optimized [excitation (collection)] spot size of $\sim 1 \mu \mathrm{m}$. A $2 \times 2$ fiber coupler delivers the light into the microscope from a Ti:sapphire $\mathrm{CW}$ tunable laser $(30 \mathrm{GHz}$ linewidth) and carries the photoluminescence signal to the detection bench. Multichannel detection is performed with an $0.3 \mathrm{~m}$ focal length double spectrograph and a backilluminated cooled Si CCD, being the spectral resolution is $\sim 75 \mu \mathrm{eV}$. The ensemble characterization is done with a standard backscattering luminescence setup with the sample held in a close-cycle cryogenerator, which is the spot diameter around $50 \mu \mathrm{m}$ in this case.

\section{RESULTS AND DISCUSSION}

Figure 1(a) shows the $I-V$ characteristic of the device at $10 \mathrm{~K}$ for different excitation conditions. The plane rectifying behavior observed in the absence of light is strongly modified when electron-hole pairs are photogenerated in the GaAs edge. At large negative voltages, photogenerated electrons are immediately depleted by the electric field contributing to the large inverse current observed below $-1.5 \mathrm{~V}$. At the same time, holes can accumulate in the GaAs/SPSL interface to form a two-dimensional hole gas $(2 \mathrm{DHG})$ in this boundary. In this regime, electron tunneling out of the $\mathrm{QRs}$ occurs before photon emission can take place, resulting in a strong quenching of the ensemble-averaged PL. Decreasing the reverse bias, the Fermi level shifts up into the QR conduction band (CB) levels. The situation is depicted in the schematic diagram of Fig. 1(b). Here, electron tunneling in and out of the QRs occurs in equilibrium with radiative recombination and up to three bands can be resolved in the PL spectra, depending on the applied voltage [Fig. 1(c)]. Starting at $-1 \mathrm{~V}$, only $\mathrm{P} 1$ is observable at $\sim 1.3 \mathrm{eV}$. In this region, the $\mathrm{PC}$ signal increases and reaches a maximum at $-0.2 \mathrm{~V}$, just when P2 dominates the PL spectrum peaked now at $\sim 1.33 \mathrm{eV}$. Same behavior is observed between $-0.2 \mathrm{~V}$ and $0.2 \mathrm{~V}$ where a new maximum in $\mathrm{PC}$ comes along with the appearance of $\mathrm{P} 3$ at $\sim 1.36 \mathrm{eV}$. A further increase of the bias voltage to positive values is accompanied by the filling of the wetting layer (WL) electron gas at $1 \mathrm{~V}$. The corresponding PL spectrum is characterized by the appearance of the WL peak at $1.422 \mathrm{eV}$. We have repeated this experiment with increased spatial resolution by using our confocal setup. The results are represented in Fig. 2(a). Out of the initially broad PL spectra, we can now resolve a finite distribution of individual peaks, but the bands, P1-P3, and their voltage dependence, can be still recognized following the envelope of the $\mu$-PL spectra.

The electro-optical characterization offers us information about the QR electronic structure. For the excitation power densities used here $\left(<1 \mathrm{~W} / \mathrm{cm}^{2}\right)$, it is unlikely that a second photogenerated electron-hole pair could be captured in a given QR during the recombination lifetime. This is confirmed by the absence of biexciton related features in the $\mu$-PL spectra of individual QRs. However, negatively 

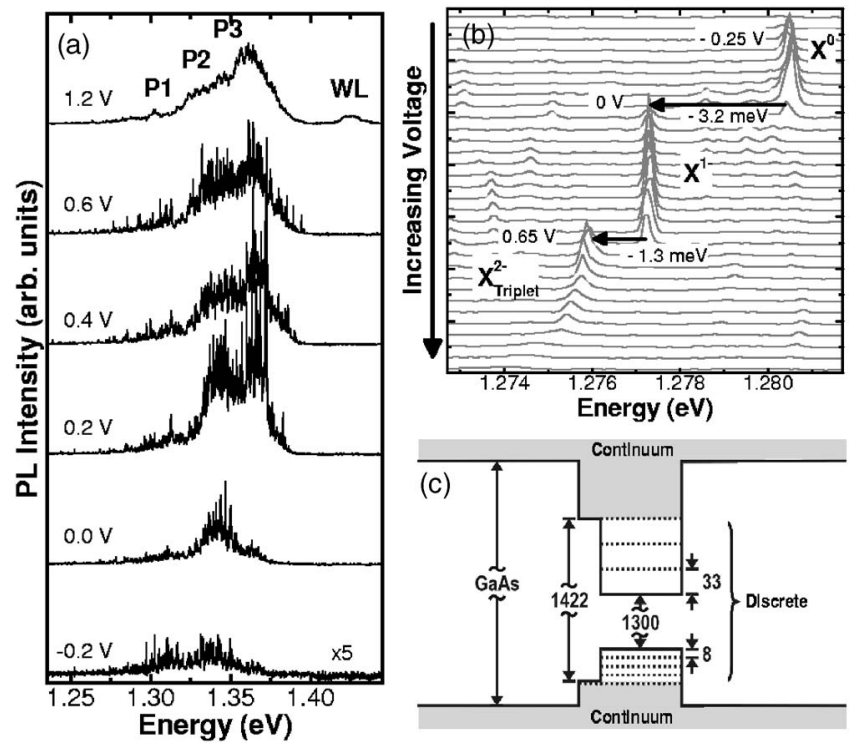

FIG. 2. (a) Voltage-dependent $\mu$-PL spectra obtained at $4.2 \mathrm{~K}$ are shown. (b) The ground state emission of a negatively charged single QR is redshifted due to the Coulomb interactions. Only the triplet $X^{2-}$ resonance could be detected with our signal to noise ratio. (c) A schematic energy level diagram for a typical QR with addition energies deduced assuming a parabolic in-plane potential.

charged excitons can be created at any time due to electron tunneling from the back contact into empty QR states. Highly charged QRs, with two or more additional electrons, have their $\mathrm{CB}$ excited states increasingly occupied. As we will discuss later, an spectator electron in the $p$ - or $d$-shell could contribute to the PL signal through direct recombination with a hole in its ground state, thus producing "hot" luminescence of charged excitons. This notwithstanding, the recombination process in the fundamental state takes place in a narrow spectral window around the neutral exciton emission line, $X^{0}$, as shown in Fig. 2(b). A sharp transition between the $X^{0}$ and $X^{1-}$ recombination occurs at $0 \mathrm{~V}$ for this QR. The emission line, now $3.2 \mathrm{meV}$ below the neutral exciton, shifts again to lower energies by $1.3 \mathrm{meV}$ when a new electron tunnels into the $\mathrm{QR}$ at $0.65 \mathrm{~V}$.

To explain these results, an estimation of the QR electronic levels including Coulomb effects is necessary. In the strong confinement regime, perturbations theory can be used to obtain the direct and exchange Coulomb contributions using the single particle states as a spanning basis. This approach is not valid anymore when the confinement is weak due to the increasing importance of correlation terms. ${ }^{32}$ With an effective 2D Bohr radius of $a_{I n A s}^{2 D} \sim 16 \mathrm{~nm}$, the first approximation seems reasonable for an InAs nucleus with only $20 \mathrm{~nm}$ diameter as observed in xSTM microscopy. In our case, to calculate the $\mathrm{QR}$ single particle energies we use a central parabolic potential which allows us to find an analytical expression for the observed splittings. The result is given by $^{33}$

$$
\Delta E_{X^{0}, X^{1-}}=\frac{e^{2} \sqrt{\pi}}{4 \pi \epsilon_{0} \epsilon_{r}}\left[\frac{1}{\sqrt{2} l_{e}}-\frac{1}{\sqrt{l_{e}^{2}+l_{h}^{2}}}\right],
$$

$$
\Delta E_{X^{1-}, X^{2-}}=\frac{e^{2} \sqrt{\pi}}{4 \pi \epsilon_{0} \epsilon_{r}}\left[\frac{3}{4 \sqrt{2} l_{e}}-\frac{2 l_{e}^{2}+l_{h}^{2}}{2\left(l_{e}^{2}+l_{h}^{2}\right)^{3 / 2}} \pm \frac{1}{4 \sqrt{2} l_{e}}\right]
$$

where the plus (minus) sign stands for the $X^{2-}$ triplet (singlet) state and $l_{e}\left(l_{h}\right)$ represents the $2 \mathrm{D}$ confinement radius for electrons (holes). Substituting the experimental values and $\epsilon_{r}^{I n A s}=15.5$, we find $l_{e} \sim 8.8 \mathrm{~nm}$ and $l_{h} \sim 4.8 \mathrm{~nm}$. Whereas, the values are slightly bigger, $l_{e} \sim 10.5 \mathrm{~nm}$ and $l_{h}$ $\sim 5.8 \mathrm{~nm}$, if we use the GaAs dielectric constant value, $\epsilon_{r}^{\text {GaAs }}=12.9$. Although the agreement with the $\mathrm{QR}$ lateral dimensions is good, it should be noted that the central parabolic model is a rough estimation for the QR potential as the role of the inner hole is completely neglected. As mentioned above, a certain softening is expected due to the incomplete dewetting process, however, the results obtained by intraband magneto-dependent spectroscopy ${ }^{12}$ cannot be explained without an electron wave function that preserves the ring geometry. On the other hand, no evidence of excitonic $A B$ oscillations have been observed in this kind of $\mathrm{QR},{ }^{14}$ a fact that could be related to an increased dotlike character of the exciton wave function due to the enhanced Coulomb interaction in small QRs. ${ }^{19,20}$ A detailed calculation of the QR electronic structure, including direct and exchange $e-h$ interactions and the potential geometry revealed by xSTM, is out of the scope of this work; therefore we use the present model with the limitations just mentioned.

Lens-shaped QDs are well described by the central parabolic model, consistent with the constant separation between electronic shells occurring in these nanostructures. ${ }^{33,34}$ In ensemble absorption experiments performed in self-assembled QRs such constant separation has been also reported with a typical splitting of $\sim 30 \mathrm{meV} .{ }^{15}$ This is exactly what we observe in our voltage-dependent PL and $\mu$-PL spectra of Figs. 1(c) and 2(a). In the framework of the parabolic model, the subshell confinement energies can be calculated from the effective carrier length using $E_{e(h)}=\hbar^{2} / m_{e(h)}^{*} l_{e(h)}^{2}$. Substituting the nominal electron effective masses for InAs and GaAs; $m_{e}^{*}=0.023$ and $m_{e}^{*}=0.067$, overestimates $(43 \mathrm{meV})$ or underestimates $(10 \mathrm{meV})$, respectively, the observed splitting. This is not surprising since InAs/GaAs alloying, together with the built-in strain field, will produce an increase of the InAs effective mass that necessarily has to be introduced ad hoc in our phenomenological model. ${ }^{27,33}$ Introducing $m_{e}^{*} \sim 0.03$ and $m_{h}^{*}=0.41$ and the confinement lengths for InAs, the corresponding addition energies $E_{e(h)}=33(8) \mathrm{meV}$ can reasonably describe our experimental results.

We assume a 70:30 band-offset partition to tentatively draw the energy scheme shown in Fig. 2(c). Only three subshells are confined in the $\mathrm{CB}$ while at least one more is available for the confined holes. Above them, the WL edge at $1.422 \mathrm{eV}$ separates the continuum from the discrete QR electronic structure. Excitonic transitions above the ground state in single QDs have been studied using $\mu$-PLE (Refs. 7 and 35-38) and direct absorption ${ }^{39}$ experiments. In our case, we use the former recording the $\mu$-PL spectra at $V_{g}=0.2 \mathrm{~V}$ of single QRs while scanning the excitation wavelength from the GaAs edge down to the ground state emission line. At this voltage, most of the studied QRs are empty or singly 


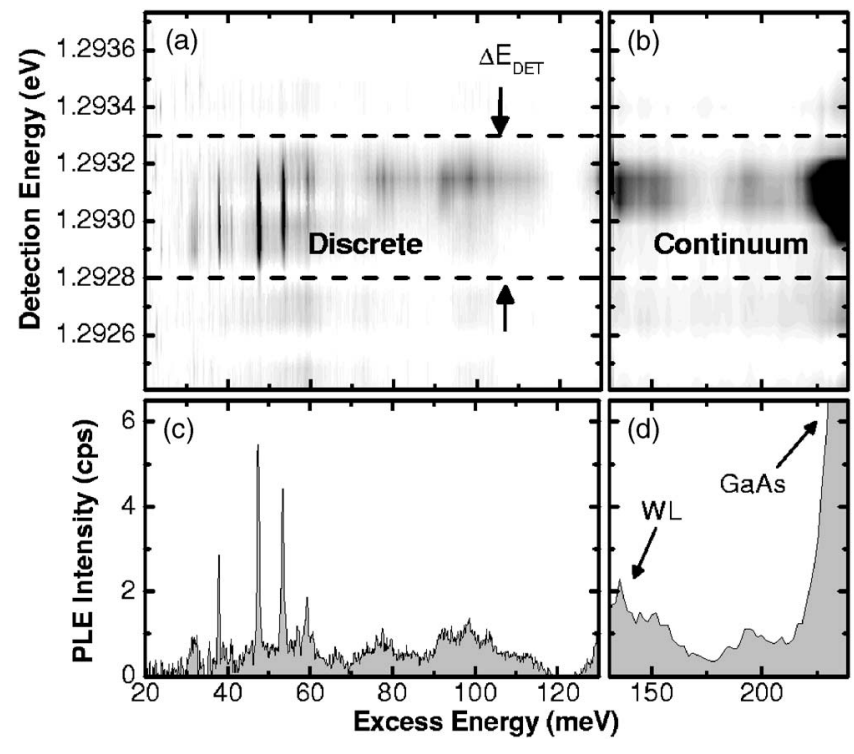

FIG. 3. Excitation spectrum images of a single $Q R$ at $V_{G}=0.2 \mathrm{~V}$ are depicted in the upper panels (left and right spectra were recorded with different resolutions, $0.1 \mathrm{~nm} / \mathrm{step}$ and $1 \mathrm{~nm} / \mathrm{step}$, respectively). Averaging over $\Delta E_{D E T}$, we obtain the $\mu$-PLE spectra shown in the lower panels.

charged before the photon absorption takes place. In this manner, we obtain a multichannel $\mu$-PLE image like the one shown in the upper panels of Fig. 3. The corresponding single channel spectrum (lower panels) can be obtained averaging over $\Delta E_{D E T}$, roughly, the spectral resolution of our spectrograph.

Due to the chromaticity of our setup, we optimized the throughput and spot size only for the detection wavelength. Nevertheless, enough intensity is available to detect spectral features corresponding to the discrete and continuum parts of the spectrum. As expected, the continuum is dominated by the WL resonance contribution as well as the GaAs barrier absorption edge [Figs. 3(b) and 3(d)]. More interesting, however, is the analysis of the discrete spectrum of several QRs represented in Figs. 3(c) and 4. It follows from the diagram in Fig. 2(c) and from the angular momentum selection rule, $m_{e}+m_{h}=0$, that the allowed single particle optical transitions are $|0,0\rangle_{e}|0,0\rangle_{h}$ in the $s$ shell, $\left(|1,-1\rangle_{e}|1,1\rangle_{h},|1,1\rangle_{e}|1,-1\rangle_{h}\right)$ in the $p$ shell, $\left(|2,-2\rangle_{e}|2,2\rangle_{h},|2,2\rangle_{e}|2,-2\rangle_{h},|2,0\rangle_{e}|2,0\rangle_{h}\right)$ in the $d$ shell, and those involving different subshells, namely, $|0,0\rangle_{e}|2,0\rangle_{h}$ and $|2,0\rangle_{e}|0,0\rangle_{h}$, all them doubly degenerated in spin. Their expected excess energies are $0 \mathrm{meV}$ for the $s$ shell, $41 \mathrm{meV}$ for the $p$ shell, $82 \mathrm{meV}$ for the $d$ shell, and $16 \mathrm{meV}$ and $66 \mathrm{meV}$ for the transitions involving unpaired shells. Comparing with our $\mu$-PLE spectra we notice that most of the sharp resonances appear in the range $\Delta E \sim 35-60 \mathrm{meV}$. Nevertheless, individual peaks can also be observed in some of the spectra at $\Delta E \sim 75-90 \mathrm{meV}$. It is well known that subshell degeneracies are lifted by the Coulomb interactions, and also by the crystal lattice and/or confining potential inplane asymmetry. QRs are nearly round objects with a small elongation along the $[1 \overline{1} 0]$ crystal direction. ${ }^{17}$ Therefore, we do not expect a large splitting between the $p_{x}$ and $p_{y}$ states.

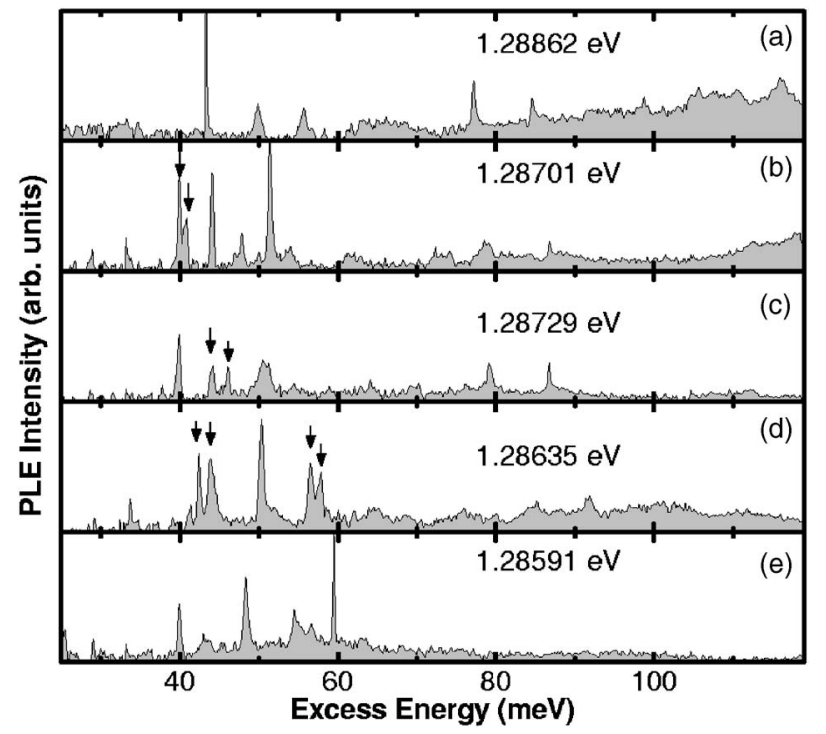

FIG. 4. Several excitation spectra of single QRs obtained in the spectral region below the WL edge are shown. Together with the sharp resonances coming from discrete optical transitions, an increasing continuous background is observed in some cases for excess energies $\Delta E>40-70 \mathrm{meV}$.

This is the case of the spectra in Figs. 4(b)-4(d) where doublet structures with energy splittings $<2 \mathrm{meV}$ are often observed between 39 an $46 \mathrm{meV}$. In other cases, the doublet structures are not present and the number of peaks is also smaller, probably indicating even more symmetric QRs. This is in contrast to InGaAs QDs where $p_{x}-p_{y}$ energy splittings larger than $5 \mathrm{meV}$ have been reported. ${ }^{40}$

A comparison between the ensemble and microscopic results will allow a better assignation to known optical transitions of the PL bands observed in Fig. 1(c). This is done in Fig. 5 on the basis of the energy scheme of Fig. 2(c) with $\Delta E=0 \mathrm{meV}$ for the ground state. We notice that according to our estimations, $\mathrm{P} 2$ centered at $\Delta E \sim 30 \mathrm{meV}$ is too close to the ground state $(\mathrm{P} 1)$ to belong to a $p$ - $p$ recombination chan-

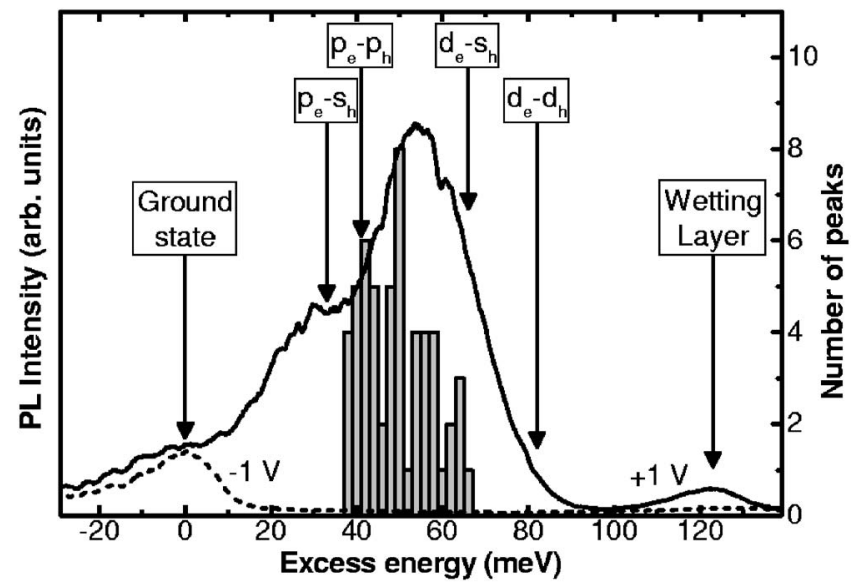

FIG. 5. The ensemble-averaged emission bands are compared with statistics performed in several $\mu$-PLE spectra (gray bars). The corresponding optical transitions are labeled according to the calculations discussed in the text. 
nel. It can be also discarded because no holes are present in the valence band excited states at low excitation powers. The same can be said for P3 that centered at $60 \mathrm{meV}$ is well above the $p-p$ transition, while still well below for being attributed to a $d$ - $d$ recombination. Our results suggest that these bands could be related to "hot" luminescence of charged excitons as mentioned above. In $\mu$-PLE, most of the peaks appeared between 35 and $70 \mathrm{meV}$. Adding the peaks found in that range in ten different QRs we can construct the bar diagram embedded in Fig. 5. The resulting distribution roughly reproduces the ensemble PL band in this range, although a major presence of the $p-p$ absorption channel is clear. The remaining resonances, found at higher energies, can be attributed to $d-d$ transitions. They are masked by the high energy tail of our emission band, but have been observed in absorption experiments done in similar samples once the WL contribution is subtracted. ${ }^{41}$

At the moment, the mechanism responsible for the observed enhancement of the luminescence coming from unpaired electronic shells is unknown to us. Ensemble absorption experiments in QRs and $\mu$-PLE spectra of QDs also exhibit such "forbidden" transitions, suggesting a violation of the interband selection rules. ${ }^{7,15,42}$ Often attributed to a strong reduction of the QD lateral symmetry, we have not found large splittings between $p_{x}$ and $p_{y}$ excited states to support such assessment. The strong polarization response of our fiber-based setup prevented us from a study of the excited states polarization properties or the magnitude of the anisotropic exchange splitting in the ground state, ${ }^{43}$ which would allow for a better determination of the QR asymmetry. Therefore, we can only conclude that the resonant injection of electrons into CB excited states is strongly correlated with the appearance of the different PL bands and explains phenomenologically our observations, yet, an argument circumventing the weak oscillator strength expected for these transitions is still missing. A more systematic study would be necessary to arrive at a satisfactory conclusion on this point.
Finally, we notice the presence of a certain background absorption below the WL edge in our $\mu$-PLE spectra (Figs. 3 and 4). Similar observations have been reported by almost any group studying the $\mu$-PLE of small QDs. ${ }^{7,35,37,38}$ Its origin has recently been assigned to bound to continuum transitions associated to the interaction between the localized and delocalized electronic polarization. ${ }^{44}$ For the electronic level structure proposed here, we expect an increasing contribution of such crossed transitions starting at an excess energy equal to the valence band offset, ${ }^{7} \Delta V_{h} \sim 40 \mathrm{meV}$. This is corroborated by our experiments, although it should be noted that its relative importance and position changes in different QRs.

\section{CONCLUSIONS}

To conclude, we have determined experimentally the excitation spectrum of single self-assembled QRs at zero magnetic field. The central parabolic model has been sufficient to describe the main features of our $\mu$-PL and $\mu$-PLE spectra and, for the aim of this study, no distinct signatures of the particular ring-shape potential could be identified. We have found that optical transitions among electronic shells of unpaired symmetry are favored by the presence of a nearby Fermi sea. Also, crossed transitions between localized and delocalized electronic states could be necessary to explain some of our results. These phenomena are beyond the artificial atom picture and depict for QRs, such as for QDs studied before, a complex scenario of strongly coupled discrete and continuum electronic polarizations.

\section{ACKNOWLEDGMENTS}

The authors would like to thank K. Karrai and R.J. Warburton for helpful discussions. This work was supported by Spanish MEC through NANOSELF Project (No. TIC200204096) and European Commission through SANDIE Network of Excellence (No. NMP4-CT-2004-500101).
*Electronic address: benito@imm.cnm.csic.es

${ }^{1}$ D. Bimberg, M. Grundmann, and N. N. Ledentsov, in Quantum Dot Heterostructures, 1st ed. (Wiley, New York, 1999).

${ }^{2}$ Single Quantum Dots, edited by P. Michler, Topics in Appied Physics Vol. 90 (Springer, Berlin, 2003).

${ }^{3}$ D. Loss and D. P. DiVincenzo, Phys. Rev. A 57, 120 (1998).

${ }^{4}$ A. Imamoglu, D. D. Awschalom, G. Burkard, D. P. DiVincenzo, D. Loss, M. Sherwin, and A. Small, Phys. Rev. Lett. 83, 4204 (1999).

${ }^{5}$ X. Li, Y. Wu, D. Steel, D. Gammon, T. H. Stievater, D. S. Katzer, D. Park, C. Piermarocchi, and L. J. Sham, Science 301, 809 (2003).

${ }^{6}$ Z. Yuan, B. E. Kardynal, R. M. Stevenson, A. J. Shields, C. J. Lobo, K. Cooper, N. S. Beattie, D. A. Ritchie, and M. Pepper, Science 295, 102 (2002).

${ }^{7}$ R. Oulton, J. J. Finley, A. I. Tartakovskii, D. J. Mowbray, M. S. Skolnick, M. Hopkinson, A. Vasanelli, R. Ferreira, and G. Bas- tard, Phys. Rev. B 68, 235301 (2003).

${ }^{8}$ K. Karrai, R. J. Warburton, C. Schulhauser, A. Höegele, B. Urbaszek, E. J. McGhee, A. O. Govorov, J. M. García, B. D. Gerardot, and P. M. Petroff, Nature (London) 427, 135 (2004).

${ }^{9}$ J. M. Smith, P. A. Dalgarno, R. J. Warburton, A. O. Govorov, K. Karrai, B. D. Gerardot, and P. M. Petroff, Phys. Rev. Lett. 94, 197402 (2005).

${ }^{10}$ O. Verzelen, R. Ferreira, and G. Bastard, Phys. Rev. Lett. 88, 146803 (2002)

${ }^{11}$ R. J. Warburton, C. Schäflein, D. Haft, F. Bickel, A. Lorke, K. Karrai, J. M. García, W. Schoenfeld, and P. M. Petroff, Nature (London) 405, 926 (2000).

${ }^{12}$ A. Lorke, R. J. Luyken, A. O. Govorov, J. P. Kotthaus, J. M. García, and P. M. Petroff, Phys. Rev. Lett. 84, 2223 (2000).

${ }^{13}$ R. J. Warburton, C. Schulhauser, D. Haft, C. Schäflein, K. Karrai, J. M. García, W. Schoenfeld, and P. M. Petroff, Phys. Rev. B 65 , 113303 (2002). 
${ }^{14}$ D. Haft, C. Schulhauser, A. O. Govorov, R. J. Warburton, K. Karrai, J. M. García, W. Schoenfeld, and P. M. Petroff, Physica E (Amsterdam) 13, 165 (2002).

${ }^{15}$ H. Pettersson, R. J. Warburton, A. Lorke, K. Karrai, J. P. Kotthaus, J. M. García, and P. M. Petroff, Physica E (Amsterdam) 6, 510 (2000).

${ }^{16}$ F. Pulizzi, D. Walker, A. Patanè, L. Eaves, M. Henini, D. Granados, J. M. García, V. V. Rudenkov, P. C. M. Christianen, J. C. Maan, P. Offermans, P. M. Koenraad, and G. Hill, Phys. Rev. B 72, 085309 (2005).

${ }^{17}$ D. Granados and J. M. García, Appl. Phys. Lett. 82, 2401 (2003).

${ }^{18}$ R. A. Römer and R. E. Raikh, Phys. Rev. B 62, 7045 (2000).

${ }^{19}$ J. Song and S. E. Ulloa, Phys. Rev. B 63, 125302 (2001).

${ }^{20}$ H. Hu, J.-L. Zhu, D.-J. Li, and J.-J. Xiong, Phys. Rev. B 63, 195307 (2001).

${ }^{21}$ I. Galbraith, F. J. Braid, and R. J. Warburton, Phys. Status Solidi A 190, 781 (2002).

${ }^{22}$ M. Bayer, M. Korkusinski, P. Hawrylak, T. Gutbrod, M. Michel, and A. Forchel, Phys. Rev. Lett. 90, 186801 (2003).

${ }^{23}$ J. M. Llorens, C. Trallero-Giner, A. García-Cristobal, and A. Cantarero, Phys. Rev. B 64, 035309 (2001).

${ }^{24}$ O. Voskoboynikov, Y. Li, H.-M. Lu, C.-F. Shih, and C. P. Lee, Phys. Rev. B 66, 155306 (2002).

${ }^{25}$ J. A. Barker, R. J. Warburton, and E. P. O'Reilly, Phys. Rev. B 69, 035327 (2004).

${ }^{26}$ J. I. Climente, J. Planelles, and F. Rajadell, J. Phys.: Condens. Matter 17, 1573 (2005).

${ }^{27}$ J. I. Climente, J. Planelles, and W. Jaskólski, Phys. Rev. B 68, 075307 (2003).

${ }^{28}$ A. Emperador, M. Pi, M. Barranco, and A. Lorke, Phys. Rev. B 62, 4573 (2000).

${ }^{29}$ A. Puente and L. Serra, Phys. Rev. B 63, 125334 (2001).

${ }^{30}$ H. Hu, G.-M. Zhang, J.-L. Zhu, and J.-J. Xiong, Phys. Rev. B 63, 045320 (2001).
${ }^{31}$ P. Offermans, P. M. Koenraad, J. H. Wolter, D. Granados, J. M. García, V. M. Fomin, V. N. Gladilin, and J. T. Devreese, Appl. Phys. Lett. 87, 131902 (2005).

${ }^{32}$ J. Shumway, A. Fraceschetti, and A. Zunger, Phys. Rev. B 63, 155316 (2001).

${ }^{33}$ R. J. Warburton, B. T. Miller, C. S. Dürr, C. Bödefeld, K. Karrai, J. P. Kotthaus, G. Medeiros-Ribeiro, P. M. Petroff, and S. Huant, Phys. Rev. B 58, 16221 (1998).

${ }^{34}$ M. Bayer, O. Stern, P. Hawrylak, S. Fafard, and A. Forchel, Nature (London) 405, 923 (2000).

${ }^{35}$ Y. Toda, O. Moriwaki, M. Nishioka, and Y. Arakawa, Phys. Rev. Lett. 82, 4114 (1999).

${ }^{36}$ P. Hawrylak, G. A. Narvaez, M. Bayer, and A. Forchel, Phys. Rev. Lett. 85, 389 (2000).

${ }^{37}$ A. Lemaitre, A. D. Ashmore, J. J. Finley, D. J. Mowbray, M. S. Skolnick, M. Hopkinson, and T. F. Krauss, Phys. Rev. B 63, 161309(R) (2001).

${ }^{38}$ C. Kammerer, G. Cassabois, C. Voisin, C. Delalande, P. Roussignol, and J. M. Gerard, Phys. Rev. Lett. 87, 207401 (2001).

${ }^{39}$ B. Alén, F. Bickel, K. Karrai, R. J. Warburton, and P. M. Petroff, Appl. Phys. Lett. 83, 2235 (2003).

${ }^{40}$ J. J. Finley, P. W. Fry, A. D. Ashmore, A. Lemaitre, A. I. Tartakovskii, R. Oulton, D. J. Mowbray, M. S. Skolnick, M. Hopkinson, P. D. Buckle, and P. A. Maksym, Phys. Rev. B 63, 161305(R) (2001).

${ }^{41} \mathrm{~K}$. Karrai (private communication).

${ }^{42}$ R. Oulton, A. I. Tartakovskii, A. Ebbens, J. Cahill, J. J. Finley, D. J. Mowbray, M. S. Skolnick, and M. Hopkinson, Phys. Rev. B 69, 155323 (2004).

${ }^{43}$ A. Högele, S. Seidl, M. Kroner, K. Karrai, R. J. Warburton, B. D. Gerardot, and P. M. Petroff, Phys. Rev. Lett. 93, 217401 (2004).

${ }^{44}$ A. Vasanelli, R. Ferreira, and G. Bastard, Phys. Rev. Lett. 89, 216804 (2002) 